SHORT REPORT

\title{
An audit of symptom triggered chlordiazepoxide treatment of alcohol withdrawal on a medical admissions unit
}

\author{
R Hardern, A V Page
}

Emerg Med J 2005;22:805-806. doi: 10.1136/emj.2004.017236

Patients suffering from alcohol withdrawal were found on initial audit to be discharged before the resolution of their symptoms. A regimen using symptom triggered chlordiazepoxide was introduced and found, on re-audit and anecolotally, to be an improvement. The duration of symptoms was reduced significantly, the length of stay was reduced (although not statistically significant because of limited power), and most patients who had experience of both regimens found the symptom triggered regimen more effective.

A medical admissions unit (MAU) admits most patients with acute medical problems, including patients with symptomatic alcohol withdrawal. Previous experience on our MAU was that these patients were often agitated or aggressive or both, that they were discharged (or selfdischarged) from hospital before resolution of symptoms, and that there was poor control of withdrawal symptoms. A treatment that reduced patients' symptoms, reduced aggression and violence, and reduced length of stay without jeopardising clinical care would be ideal.

\section{METHODS}

A decision was taken by our MAU staff to keep patients admitted because of alcohol withdrawal on the MAU until they were fit for discharge. The senior nursing staff, consultant, and pharmacist agreed that efforts should be made to improve the care of patients with alcohol withdrawal, and that a regimen described previously ${ }^{1-3}$ should be piloted. The trial ${ }^{1}$ on which this decision was based excluded patients with a history of fits; although such patients may be expected to benefit most from improved treatment of alcohol withdrawal, we chose, for the purposes of the trial period, to adopt the same exclusion criteria. We chose to treat excluded patients with regular, rather than symptom triggered, chlordiazepoxide (as was normal practice before the pilot).

Since training in the use of the scoring instrument (Clinical Institute Withdrawal Assessment for Alcohol, revised (CIWAAr)) was not provided to wards other than the MAU, its use was limited to the MAU. We identified patients admitted because of alcohol withdrawal from a log kept on the ward and by searching discharge summaries. (This included patients treated before the introduction of symptom triggered chlordiazepoxide. Their notes were reviewed retrospectively.) As the numbers in each group were small, we performed nonparametric comparisons between the two groups (two tailed Mann-Whitney U test).

\section{RESULTS}

Data were available on 51 patients, 23 of whom were managed according to the symptom triggered regimen. Some patients received regular treatment because of previous fits, others because some medical staff were unfamiliar with the symptom triggered regimen, and some because (in the early phase) there were no nurses on duty who had been trained in the use of the scoring tool. No fits were seen in any patient.

\section{Total dose}

Because published data suggested that symptom triggered treatment may lead to a lower overall dose being used, we compared the groups with regard to dose. The median total dose of chlordiazepoxide was $600 \mathrm{mg}$ in the symptom triggered (ST) group $(\mathrm{n}=23)$ and $607.5 \mathrm{mg}$ in the regular group $(\mathrm{n}=28)(\mathrm{p}=0.31)$.

\section{Length of stay}

Length of stay recorded was the number of whole days the patient spent in hospital (including period waiting for admission to the MAU). Discharge times are not always recorded, so this may not be entirely accurate. The median length of stay was 3 days in the ST group $(n=23)$ and 5.5 days in the regular group $(\mathrm{n}=28)(\mathrm{p}=0.086)$.

\section{Interval between first and last dose of chlordiazepoxide}

The median interval was 48 hours in the ST group $(\mathrm{n}=23$ ) and 110 hours in the regular group $(n=28)$. This interval was significantly shorter in the symptom triggered group $(\mathrm{p}=0.0018)$.

No formal qualitative assessment was made, but most patients found the symptom triggered regimen acceptable (some who had previously received regular treatment preferred the symptom triggered regimen) even though some found it tiresome to be assessed every hour (when not asleep). Nursing staff felt the symptom triggered regimen led to fewer episodes of patient agitation or aggression and consequently wanted to continue with this treatment after the pilot period.

\section{DISCUSSION}

The audit data presented above support previous trial data that use of symptom triggered chlordiazepoxide leads to more rapid resolution of symptoms from alcohol withdrawal. The audit had limited statistical power because of the small number of patients involved. The difference in median length of stay would probably have been statistically significant with more patients; certainly the crude difference in median of 2.5 days would be clinically (and economically) important. No increase in the frequency of fits was seen. The regimen proved acceptable to most patients and nursing staff, and the latter were reluctant to discontinue the pilot of the symptom triggered regimen. Although it did entail hourly assessment of patients (this could be discontinued once scores had been low for eight hours), the benefits to the staff (primarily a marked reduction in aggressive or violent behaviour among 


\section{Key points}

- The use of symptom triggered chlordiazepoxide to treat alcohol withdrawal is feasible on a busy medical admissions unit.

- With this regimen the overall dose given to patients does not change but, because it tends to be given over a shorter period, symptoms are relieved more rapidly and duration of treatment is shorter.

- Anecdotal experience was that the symptom-triggered regimen is associated with fewer episodes of violence or aggression.

patients) outweighed this extra work. With experience the scoring did not take more than a few minutes to complete. We did not find this flexible regimen impractical, despite being a busy general ward. ${ }^{4}$

\section{ACKNOWLEDGEMENTS}

$\mathrm{R}$ Frampton, J-M Carter, and J Widdowson helped with data collection. R Frampton helped teach nursing staff how to use this approach to manage alcohol withdrawal. The benefits of this system would not have been found without the hard work and cooperation of the nursing and medical staff on the Medical Admissions Unit.

\section{Authors' affiliations}

R Hardern, A V Page, Medical Admissions Unit, The General Infirmary, Leeds, UK

Competing interests: none declared

Correspondence to: Dr R D Hardern, Accident and Emergency, University Hospital of North Durham, North Road, Durham, DH1 5TW; richard.hardern@cddah.nhs.uk

Accepted for publication 20 September 2004

\section{REFERENCES}

1 Saltz R, Mayo-Smith MF, Roberts MS, et al. Individualized treatment for alcohol withdrawal: a randomized double-blind controlled trial. JAMA 1994;272:519-23.

2 Sullivan JT, Sykora K, Schneiderman J, et al. Assessment of alcohol withdrawal: the revised Clinical Institute Withdrawal Assessment for Alcohol scale (CIWA-Ar). Br J Addict 1989;84:1353-7.

3 Mayo-Smith MF, Cushman P, Hill AJ, et al. Pharmacological management of alcohol withdrawal: a meta-analysis and evidence-based practice guideline. JAMA 1997;278:144-51

4 Welch S. Management of substance misuse problems in the general hospital. Clin Med 2002;2:513-15. 